\title{
Immunological Studies in Kaposi's Sarcoma in Uganda
}

\author{
S. P. MASTER, ${ }^{*}$ M.B., B.S., F.R.C.S. ; J. F. TAYLOR, $†$ M.D., D.T.M.\&H., F.R.C.S.
}

S. K. KYALWAZI, $\ddagger$ M.B., CH.B., F.R.C.S.ED. ; J. L. ZIEGLER, $\$ M.D.

\begin{abstract}
Summary: An evaluation of humoral and cellular $\int$ immune mechanisms was performed on patients with Kaposi's sarcoma in Uganda. Antibody responses and immunoglobulin levels were normal in all patients studied. Nevertheless, a striking impairment in the delayed hypersensitivity response to dinitrochlorobenzene was noted in patients with the "malignant" type of tumour.
\end{abstract}

\section{Introduction}

Kaposi (1894) first described the clinical and pathological features of "sarcoma idiopathic multiplex haemorrhagicum." Today the histological features and the clinical manifestations of Kaposi's sarcoma are still in the descriptive phase, and basic information regarding the possible aetiology, origin, and nature of the neoplastic cell, immunology, and response to therapy is scant.

The high incidence of Kaposi's sarcoma in Uganda provides a unique opportunity to investigate its clinical and pathological features. In a recent study of the clinical appearance and treatment of Kaposi's sarcoma in Uganda, Kyalwazi (1969) delineated two major types: "benign" and "malignant." Patients with the benign type present with a long history of subcutaneous nodules or raised hyperpigmented plaques on the extremities, often with oedema of the involved limb. Patients with the malignant type usually develop invasive deep ulcerating tumours or lymph node, visceral, and bone involvement. Some patients show a transition from the benign to the malignant type of lesion during their clinical course. This classification has been useful in the past in predicting the response to chemotherapy and the prognosis of the disease (Kyalwazi, 1968).

Some clinical and histological features of Kaposi's sarcoma suggest that host immunity may influence the clinical manifestation and progression of the disease. The evidence for this hypothesis includes the indolent and benign nature of the clinical course in many cases (Kyalwazi, 1968), the documented occurrence of spontaneous remissions (Lothe, 1963; Cook, 1966), and the infiltration of the tumour by lymphocytes and plasma cells (Lothe, 1963; Slavin et al., 1969). Accordingly, an evaluation of humoral and cellular immunological responses in Ugandan patients with Kaposi's sarcoma was performed.

\section{Selection of Patients}

The patients studied were admitted to the surgical wards of the New Mulago Hospital, Kampala, Uganda, between Sep-

* Senior Registrar, Mulago Hospital, Kampala, Uganda.

† Lecturer, Department of Surgery, Makerere University Medical School, Kampala, Uganda.

¥ Professor of Surgery, Makerere University Medical School, Kampala, Uganda.

$\checkmark$ Director, Lymphoma Treatment Centre, Kampala, Uganda; Senior Investigator, Medicine Branch, National Cancer Institute, Bethesda, Maryland. tember 1967 and April 1969. All had a histological diagnosis of Kaposi's sarcoma from the department of pathology, Makerere University Medical School, Kampala. The patients were evaluated clinically and placed into one of two categories: benign or malignant. The appearance of the lesion in patients with these two clinical manifestations is shown in Fig. 1 and 2. Immunological studies were made on untreated

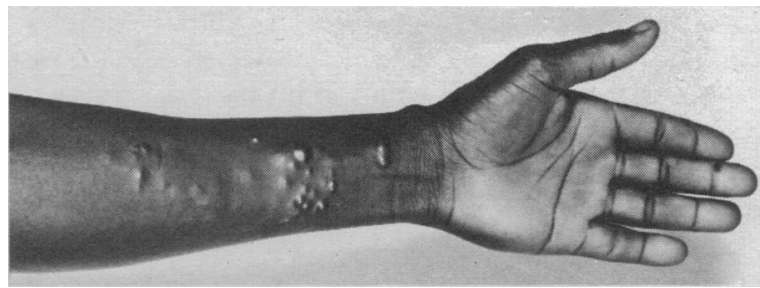

FIG. 1.-Showing lesion in a benign type of Kaposi's sarcoma.

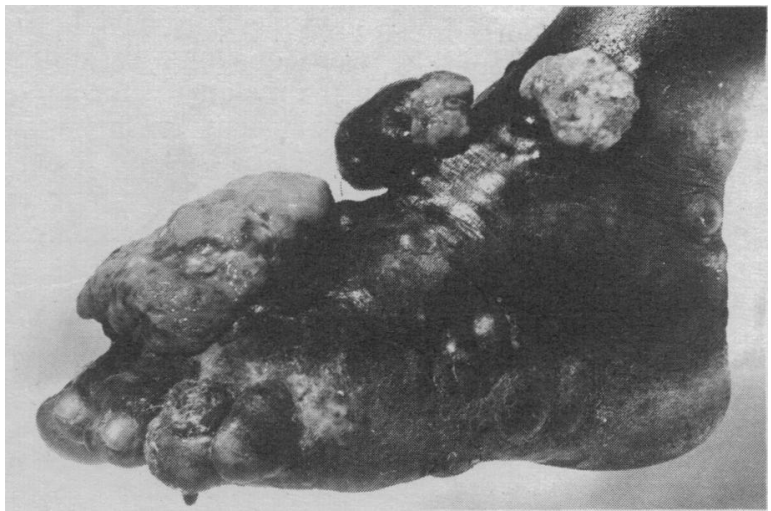

FIG. 2.-Showing lesion in a malignant type of Kaposi's sarcoma.

patients under 50 years of age. Patients who were on chemotherapy or who had concurrent debilitating diseases were excluded from the study. All immunological testing procedures were approved for clinical investigation by research committees at the National Cancer Institute, Bethesda, Maryland, and at Makerere University Medical School and Mulago Hospital, Kampala, Uganda. All patients and control subjects gave their consent to the procedures.

\section{Immunological Evaluation}

Humoral antibody response to Escherichia coli Vi antigen (kindly supplied by Dr. M. E. Webster, National Heart Institute, Bethesda, Md.) was evaluated by determining the antibody titre rise two weeks after an intramuscular immunizing dose of $100 \mu \mathrm{g}$. Vi antibody titres were determined in twofold dilutions by a haemagglutination technique (Landy and Lamb, 1953). 
$\mathrm{Vi}$ antibody responses were determined in 14 patients with the benign type of Kaposi's sarcoma, and in six patients with the malignant type. Seventeen men in hospital with trauma or minor surgery served as control subjects.

Immunoglobulin levels were performed by the gel-diffusion method (Fahey and McKelvey, 1965) through the courtesy of Dr. Martin H. Cohen, of the National Cancer Institute, Eethesda, Md. Sera from six patients with benign and four with malignant Kaposi's sarcoma were tested. Control Ig determinations were derived from the sera of 12 normal African adults. The sera used for Ig determinations were the preimmunization specimens from patients receiving $\mathrm{Vi}$ antigen.

Delayed hypersensitivity responses were measured using dinitrochlorobenzene sensitization and subsequent challenge according to the method of Brown et al. (1967). A 2,000 $\mu$ g.-sensitizing dose of $0.1 \mathrm{ml}$. of dinitrochlorobenzene in acetone was allowed to evaporate within a $2-\mathrm{cm}$.diameter polyethylene disc on the medial aspect of the right arm and was covered for one week with an occlusive dressing. Fourteen days after the sensitizing dose, challenge doses of 100 and $50 \mu \mathrm{g}$. of dinitrochlorobenzene were similarly applied to separate sites on the right forearm and covered with occlusive dressings. These sites were examined at 48-72 hours and graded as positive if induration, vesicles, or bullae were present. Dinitrochlorobenzene testing was performed on 30 patients with the benign type and on 16 with the malignant type of Kaposi's sarcoma. Thirty adult patients under 50 years of age in hospital for trauma or minor surgery served as controls for dinitrochlorobenzene testing.

\section{Results}

A total of 60 patients (58 males and 2 females) with Kaposi's sarcoma were considered for immunological testing during in study period. Fourteen were eliminated from the final study group for the following reasons: previous chemotherapy (4), over 50 years of age (6), and died or lost to follow-up before testing completed (4). Of the final group of 46 patients 30 had benign and 16 had malignant types of Kaposi's sarcoma.
The Vi antibody titre rises in 15 patients with benign or malignant Kaposi's sarcoma who had a preimmunization titre of less than 1:8 are shown in Fig. 3. There is no difference in titres between patients with the two types of Kaposi's sarcoma, and all subjects showed normal antibody responses.

The IgG levels in 10 patients with Kaposi's sarcoma are shown in Fig. 4, all values falling within the normal range. Again no differences were observed between the benign and malignant groups.

The results of dinitrochlorobenzene testing are shown in the Table. All patients with the benign type of Kaposi's sarcoma had positive reactions. Only 2 out of 16 patients with the

Delayed Hypersensitivity in Kaposi's Sarcoma

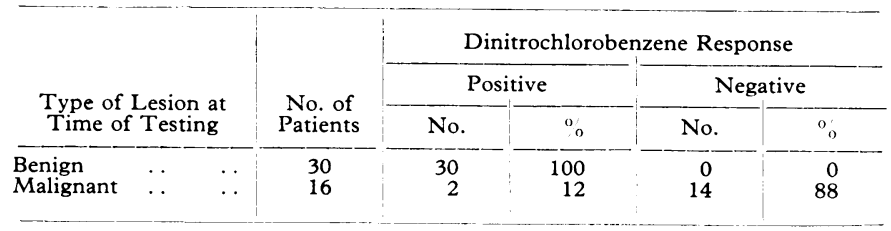

malignant type were successfully sensitized to dinitrochlorobenzene, showing a highly significant depression of delayed hypersensitivity among patients in this group $(P<0.001)$. None of these patients had extensive lymph node involvement, though local tumour infiltration of regional lymph nodes of the involved extremity was noted in some. All 30 control subjects showed a positive response to dinitrochlorobenzene.

Serial retesting of dinitrochlorobenzene reactivity was performed in a small number of patients. Six who remained in the nodular stage were rechallenged $2,3,6,6,7$, and 15 months after sensitization and remained positive despite intervening chemotherapy. Three patients with initially benign lesions and positive dinitrochlorobenzene responses progressed to the malignant stage, and challenges 1,1 , and 3 months following the transition were negative. Two of the three patients also failed to respond to dinitrochlorobenzene after resensitization procedures. Dinitrochlorobenzene responses in three patients who presented with malignant lesions remained negative on repeat tests.

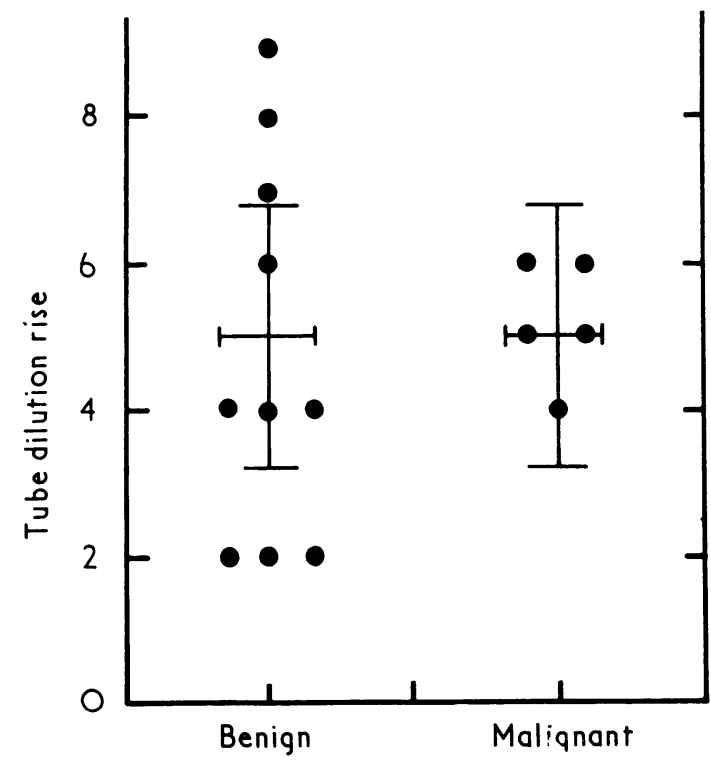

FIG. 3.- $-\mathrm{Vi}$ antibody response in Kaposi's sarcoma. Crossbars indicate mean \pm S.D. in controls.

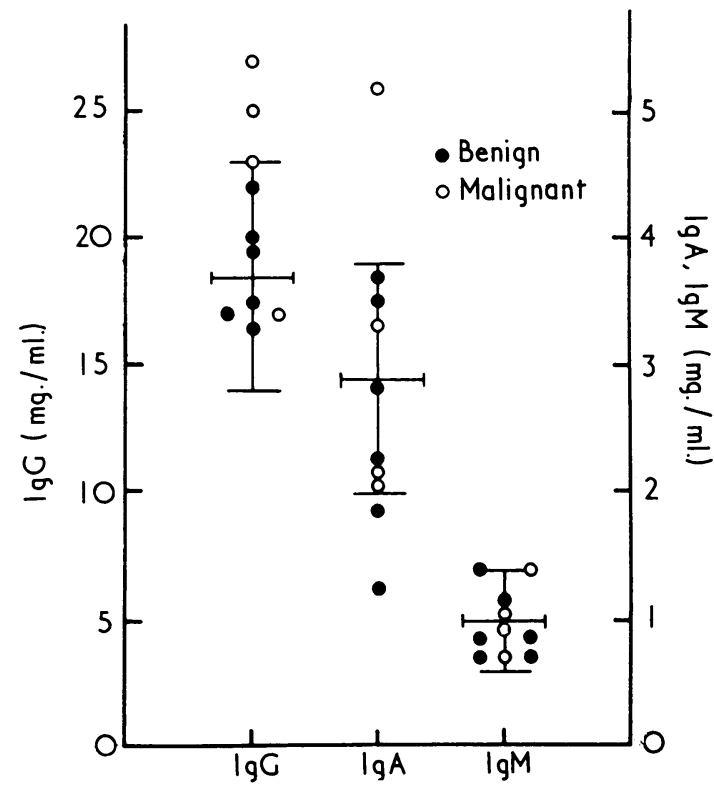

FIG. 4.-Immunoglobulin levels in Kaposi's sarcoma. Crossbars indicate mean \pm S.D. in controls. 


\section{Discussion}

Patients with Kaposi's sarcoma appear to have normal humoral immunity as measured in this study. Nevertheless, a striking impairment was noted in the delayed hypersensitivity response to dinitrochlorobenzene in those with the malignant form of the disease. Serial testing suggests that the defect in cellular immunity may accompany a malignant change in the clinical manifestation of the disease. Whether the defect is related to the pathogenesis or is the result of this malignant change remains unknown. There is evidence that patients with impaired immunity may be more susceptible to the development of neoplastic disease (Smith, 1968), and Kaposi's sarzoma has been described as arising in a patient with immunosuppression following a homograft (Siegel et al., 1969). On the other hand, some patients with advanced cancer may manifest depressed cellular immunity (Solowey et al., 1965). Further studies will be necessary fully to elucidate the dynamic nature and extent of impairment of cellular immunity in malignant Kaposi's sarcoma and the relationship of immunological status to infectious complications, response to therapy, and the histopathology of the lesions.

The finding of impaired delayed hypersensitivity in advanced Kaposi's sarcoma parallels the observations of the immune disorder in patients with Hodgkin's disease (Aisenberg, 1966; Brown, et al., 1967). Both neoplasms have appeared concurrently in the same patient in much greater than chance association (Reynolds et al., 1965; O'Brien and Brasfield, 1966). The exact pathogenetic and clinical relationships between Kaposi's sarcoma and Hodgkin's disease, however, remain speculative (Lancet, 1967).

We are aware that the distinction between the benign and malignant types of Kaposi's sarcoma is based on clinical grounds alone. In a review of 70 patients seen at Mayo Clinic, Reynolds et al. (1965) pointed out the variations in the clinical appearance of the tumour, and attempted to correlate the gross lesion with the histological features. In a large series from Tanzania (Slavin et al., 1969) both the benign and the relatively fulminant course of Kaposi's sarcoma were emphasized, though pathological correlations could not be made. From the present study there appears to be a valid immunological basis as well as a clinical basis for the division of Kaposi's sarcoma into benign and malignant types.

We wish to thank the department of medical illustration and $\mathrm{Mr}$. Cardozo, Makerere University Medical School, for Figs. 1-4 Thanks are also due to Mrs. S. Weymark, Mrs. Mary Proctor, and Miss Eleanor Stashick for technical assistance. We are grateful to Mr. D. Bhana for permission to include his patients in the study, and to Dr. R. H. Morrow for advice on the manuscript. This work was supported in part by the British Empire Cancer Campaign, the British Medical Research Council, and Contract Nos. PH 43-6747 and PH 43-67-1343 from the National Institutes of Health, United States Public Health Service, Bethesda, Md.

Address for reprints: Dr. J. L. Ziegler, Lymphoma Treatment Centre, Box 3935, Kampala, Uganda.

\section{REFERENCES}

Aisenberg, A. C. (1966.) Cancer Research, 26, 1152

Brown, R. S., et al. (1967). Annals of Internal Medicine, 67, 291.

Cook, J. (1966). Fournal of the Royal Coilege of Surgeons of Edinburgh, $11,185$.

Fahey, J. L., and McKelvey, E. M. (1965). Fournal of Immunology, 94,

Kaposi, M. (1894). Archiv für Dermatologie und Syphilis, 29, 164.

Kyalwazi, S. K. (1968). East African Medical fournal, 46, 450.

Kyalwazi, S. K. (1969. East African Medical fournal, 45, 17.

Lancet, 1967, 2, 1290.

Landy, M., and Lamb, E. (1953). Proceedings of the Society for Experimental Biology and Medicine, 82, 593.

imental Biology and Medicine, 82, 593.
Lothe, F. (1963). Kaposi's Sarcoma in Uganda Africans. Oslo, Universitetsforlaget.

O'Brien, P. H., and Brasfield, R. D. (1966). Cancer (Philadelphia), 19, 1497.

Reynolds, W. A., Winkelmann, R. K., and Soule, E. H. (1965). Medicine, 44, 419.

Siegel, J. H., et al. (1969). Fournal of the American Medical Association, $207,1493$.

Slavin, G., Cameron, H. McD., and Singh, H. (1969). British fournal of Cancer, 23, 349.

Smith, R. T. (1968). New England fournal of Medicine, 278, 1268. Solowey, A. C., and Rapaport, F. T. (1965). Surgery, Gynecology and
Obstetrics, 121, 756.

\title{
Study of Childhood Urinary Tract Infection in General Practice
}

\author{
N. C. MOND, ${ }^{*}$ M.R.C.S., L.R.C.P., M.R.C.G.P.; R. N. GRÜNEBERG, † M.B., M.R.C.PATH.
}

JEAN M. SMELLIE, $\ddagger$ B.M., M.R.C.P., D.C.H.

\begin{abstract}
Summary: A study of bacteriuria was conducted among 426 of the 436 children under the age of 13 in a general practice in north-west London. Three girls and one boy were found to have asymptomatic bacteriuria, and a further girl with bacteriuria presented with abdominal pain and fever. The calculated incidence of urinary tract infection was $1.4 \%$ per annum. Most of the childhood urinary infections in this practice occurred before the age of 5 yeans, and the incidence of significant bacteriuria in this age group was $4.9 \%$ per annum. Five other children (four girls and one boy) in the practice were known to have had proved urinary tract infection. Of the
\end{abstract}

*General Practitioner, Kingsbury, London N.W.9.

tConsultant Bacteriologist, University College Hospital, London W.C.1. $\ddagger$ Research Assistant, Department of Paediatrics, University College Hospital, Londen W.C.1. total of eight children known to have had significant bacteriuria and investigated radiologically, three girls and two boys had radiological abnormalities in the urinary tract.

Pyuria and proteinuria did not prove to be useful in the prediction of asymptomatic bacteriuria. Urinary tract infection with renal tract abnormality was found in this practice to be at least five times as common as diabetes in childhood.

\section{Introduction}

Studies of urinary infection in adults in general practice have been reported Fry et al., 1962; Loudon and Greenhalgh, 1962; Gallagher et al., 1965; Mond et al., 1965). Surveys have also been made of the prevalence of bacteriuria in schoolgirls (Kunin et al., 1964; Meadow et al., 1969; Savage et al., 1969) 